No dependence of coupling constants $f^{F}, f^{D}, f^{S}$ in vector-meson- $1 / 2^{+}$octet baryon interaction Lagrangian on the choice of the $\omega-\phi$ mixing configuration.

\title{
Cyril Adamuščín*
}

Institute of Physics, Slovak Academy of Sciences Bratislava, 845 11, Slovakia

E-mail: cyril.adamuscinesavba.sk

\section{Erik Bartoš}

Institute of Physics, Slovak Academy of Sciences Bratislava, 845 11, Slovakia

E-mail: erik.bartos@savba.sk

\section{Stanislav Dubnička}

Institute of Physics, Slovak Academy of Sciences Bratislava, 845 11, Slovakia

E-mail: stanislav.dubnicka@savba.sk

\section{Anna Zuzana Dubničková}

Depart. of Theoretical Physics, Comenius University Bratislava, 842 48, Slovakia

E-mail: dubnickova@fmph.uniba.sk

It is shown that multiply definitions of the $\omega-\phi$ mixing exist in the literature and that they seemingly lead to the different behaviors of the vector meson-baryon-antibaryon interaction. The seeming ambiguity is resolved.

The European Physical Society Conference on High Energy Physics

5-12 July

Venice, Italy

${ }^{*}$ Speaker. 
Different definitions of the $\omega-\phi$ mixing in the literature $[1,2,3]$ seemingly lead to the different expressions for the $f^{F}, f^{D}, f^{S}$ in vector-meson-1/2+ octet baryon interaction Lagrangian and therefore also to the different theoretical predictions of the electromagnetic structure of the hyperons.

The strong interaction of the vector-mesons with the $1 / 2^{+}$octet baryons is described by the $S U(3)$ invariant interaction Lagrangian

$$
\begin{aligned}
\mathscr{L}_{V B \bar{B}} & =\frac{\mathrm{i}}{\sqrt{2}} f^{F}\left[\bar{B}_{\beta}^{\alpha} \gamma_{\mu} B_{\gamma}^{\beta}-\bar{B}_{\gamma}^{\beta} \gamma_{\mu} B_{\beta}^{\alpha}\right]\left(V_{\mu}\right)_{\alpha}^{\gamma} \\
& +\frac{\mathrm{i}}{\sqrt{2}} f^{D}\left[\bar{B}_{\gamma}^{\beta} \gamma_{\mu} B_{\beta}^{\alpha}+\bar{B}_{\beta}^{\alpha} \gamma_{\mu} B_{\gamma}^{\beta}\right]\left(V_{\mu}\right)_{\alpha}^{\gamma}+\frac{\mathrm{i}}{\sqrt{2}} f^{S} \bar{B}_{\beta}^{\alpha} \gamma_{\mu} B_{\alpha}^{\beta} \omega_{\mu}^{0},
\end{aligned}
$$

where $B, \bar{B}$ are baryon, anti-baryon octet matrices and $V, \omega_{0}$ are a vector meson octet matrix and a vector meson singlet.

$$
B=\left(\begin{array}{ccc}
\frac{\Sigma^{0}}{\sqrt{2}}+\frac{\Lambda^{0}}{\sqrt{6}} & \Sigma^{+} & p \\
\Sigma^{-} & -\frac{\Sigma^{0}}{\sqrt{2}}+\frac{\Lambda^{0}}{\sqrt{6}} & n \\
\Xi^{-} & \Xi^{0} & -\frac{2 \Lambda^{0}}{\sqrt{6}}
\end{array}\right), V=\left(\begin{array}{ccc}
\frac{\rho^{0}}{\sqrt{2}}+\frac{\omega_{8}}{\sqrt{6}} & \rho^{+} & K^{+} \\
\rho^{-} & -\frac{\rho^{0}}{\sqrt{2}}+\frac{\omega_{8}}{\sqrt{6}} & K^{0} \\
K^{-} & \bar{K}^{0} & -\frac{2 \omega_{8}}{\sqrt{6}}
\end{array}\right) .
$$

The $\omega_{0}, \omega_{8}$ vector meson states do not correspond tho the physical states $\omega, \phi$, therefore $\omega-\phi$ mixing has been introduced by different authors as [1]

$$
\begin{aligned}
& \phi=\omega_{8} \cos \theta-\omega_{0} \sin \theta \\
& \omega=\omega_{8} \sin \theta+\omega_{0} \cos \theta,
\end{aligned}
$$

or [2]

$$
\begin{aligned}
& \phi=-\omega_{8} \cos \theta+\omega_{0} \sin \theta \\
& \omega=\omega_{8} \sin \theta+\omega_{0} \cos \theta,
\end{aligned}
$$

or [3]

$$
\begin{aligned}
& \phi=\omega_{8} \cos \theta+\omega_{0} \sin \theta \\
& \omega=-\omega_{8} \sin \theta+\omega_{0} \cos \theta,
\end{aligned}
$$

with $\theta \approx 39.1^{\circ}$. However, the mixing [3] is physically non-acceptable.

It can be shown that there are eight possible $\omega-\phi$ mixing versions fulfilling the Gell-MannOkubo quadratic mass formula for the vector meson, but only four of them are physically acceptable with quark representation of the $\phi$ meson to be dominated by the $s \bar{s}$ state - in the case of an ideal mixing angle $\theta \approx 35.3^{\circ}$

$$
\begin{array}{lllll}
\phi=\omega_{8} \cos \theta-\omega_{0} \sin \theta, & \omega=\omega_{8} \sin \theta+\omega_{0} \cos \theta & \Rightarrow & \phi \approx-s \bar{s}, & \omega \approx(u \bar{u}+d \bar{d}) / \sqrt{2} \\
\phi=-\omega_{8} \cos \theta+\omega_{0} \sin \theta, & \omega=\omega_{8} \sin \theta+\omega_{0} \cos \theta & \Rightarrow & \phi \approx s \bar{s}, & \omega \approx(u \bar{u}+d \bar{d}) / \sqrt{2} \\
\phi=\omega_{8} \cos \theta-\omega_{0} \sin \theta, & \omega=-\omega_{8} \sin \theta-\omega_{0} \cos \theta & \Rightarrow & \phi \approx-s \bar{s}, & \omega \approx-(u \bar{u}+d \bar{d}) / \sqrt{2} \\
\phi=-\omega_{8} \cos \theta+\omega_{0} \sin \theta, & \omega=-\omega_{8} \sin \theta-\omega_{0} \cos \theta & \Rightarrow \phi \approx s \bar{s}, & \omega \approx-(u \bar{u}+d \bar{d}) / \sqrt{2}
\end{array}
$$


The product of the $B, \bar{B}, V$ matrices in the $\mathscr{L}_{V B \bar{B}}$ Lagrangian can be expanded in order to obtain coupling constants of the particular vector meson-baryon-baryon terms as a function of the $f^{F}, f^{D}, f^{S}$ coefficients with addition of the substitution the $\omega_{0}, \omega_{8}$ states by the $\omega, \phi$. In the case of nucleons and the $\omega-\phi$ mixing [1]

$$
\begin{aligned}
& f_{\rho N N}=\frac{1}{2}\left(f^{F}+f^{D}\right) \\
& f_{\phi N N}=\frac{1}{2 \sqrt{3}}\left(3 f^{F}-f^{D}\right) \cos \theta-\frac{1}{\sqrt{2}} f^{S} \sin \theta \\
& f_{\omega N N}=\frac{1}{2 \sqrt{3}}\left(3 f^{F}-f^{D}\right) \sin \theta+\frac{1}{\sqrt{2}} f^{S} \cos \theta .
\end{aligned}
$$

The inverse relations show $f^{F}, f^{D}, f^{S}$ as functions of $f_{\rho N N}, f_{\phi N N}, f_{\omega N N}$

$$
\begin{aligned}
f^{F} & =\frac{1}{2}\left[f_{\rho N N}+\sqrt{3}\left(f_{\phi N N} \cos \theta+f_{\omega N N} \sin \theta\right)\right] \\
f^{D} & =\frac{1}{2}\left[3 f_{\rho N N}-\sqrt{3}\left(f_{\phi N N} \cos \theta+f_{\omega N N} \sin \theta\right)\right] \\
f^{S} & =\sqrt{2}\left(f_{\omega N N} \cos \theta-f_{\phi N N} \sin \theta\right)
\end{aligned}
$$

In the case of the $\omega-\phi$ mixing [2] one gets a different result

$$
\begin{aligned}
f^{F} & =\frac{1}{2}\left[f_{\rho N N}+\sqrt{3}\left(-f_{\phi N N} \cos \theta+f_{\omega N N} \sin \theta\right)\right] \\
f^{D} & =\frac{1}{2}\left[3 f_{\rho N N}-\sqrt{3}\left(-f_{\phi N N} \cos \theta+f_{\omega N N} \sin \theta\right)\right] \\
f^{S} & =\sqrt{2}\left(f_{\omega N N} \cos \theta+f_{\phi N N} \sin \theta\right) .
\end{aligned}
$$

There are yet two more sets of different expressions for the other two mixings, which may mean that there are four different predictions of the $f^{F}, f^{D}, f^{S}$ coupling constants values. However, we will show that it is not the case.

The coupling constants of the vector mesons with nucleons $f_{V N N}$ can be calculated from the ratio $\left(f_{V N N} / f_{V}\right)$ which can be extracted from the experimental data on the electromagnetic (EM) structure of the nucleons under the assumption of the vector meson dominance by means of the Unitary and Analytic model of the nucleon EM structure [4]. The absolute value of the coupling constant of a vector meson with photon $f_{V}$ can be measured separately in the leptonic decay of the vector mesons

$$
\Gamma\left(V \rightarrow e^{+} e^{-}\right)=\frac{\alpha^{2} m_{V}}{3}\left(\frac{f_{V}^{2}}{4 \pi}\right)^{-1} .
$$

The relative signs of the particular coupling constants $f_{\rho}, f_{\omega}, f_{\phi}$ depend on the chosen $\omega-$ $\phi$ mixing version Eqs.(6)-(9). In order to derive this ratio correctly one needs to compare two equations for the EM hadronic current [5]

$$
J_{\mu}^{H}=-\frac{m_{\rho}^{2}}{f_{\rho}} \rho_{\mu}-\frac{m_{\omega}^{2}}{f_{\omega}} \omega_{\mu}-\frac{m_{\phi}^{2}}{f_{\phi}} \phi_{\mu}
$$


and

$$
\begin{aligned}
J_{\mu}^{H} & =\frac{2}{3} \bar{u} \gamma_{\mu} u-\frac{1}{3} \bar{d} \gamma_{\mu} d-\frac{1}{3} \bar{s} \gamma_{\mu} s= \\
& =\frac{1}{2}\left(\bar{u} \gamma_{\mu} u-\bar{d} \gamma_{\mu} d\right)+\frac{1}{6}\left(\bar{u} \gamma_{\mu} u+\bar{d} \gamma_{\mu} d\right)-\frac{1}{3} \bar{s} \gamma_{\mu} s \\
& =\frac{1}{\sqrt{2}} J_{\mu}^{\rho^{0}}+S_{\omega} \frac{1}{3 \sqrt{2}} J_{\mu}^{\omega}-S_{\phi} \frac{1}{3} J_{\mu}^{\phi} .
\end{aligned}
$$

The latter was rewritten in terms of the vector meson currents

$$
J_{\mu}^{\rho}=\frac{1}{\sqrt{2}}\left(\bar{u} \gamma_{\mu} u-\bar{d} \gamma_{\mu} d\right), J_{\mu}^{\phi}=S_{\phi} \bar{s} \gamma_{\mu} s, J_{\mu}^{\omega}=S_{\omega} \frac{1}{\sqrt{2}}\left(\bar{u} \gamma_{\mu} u+\bar{d} \gamma_{\mu} d\right),
$$

which depend on the quark content of the particular vector meson. The quark content of the $\omega$ and $\phi$ mesons depend on the chosen $\omega-\phi$ mixing version - see the Eq.(6)-(9) for the signs $S_{\omega}, S_{\phi}$.

Now by comparing dimensionless coefficients in front of particular vector meson field/current one obtains ratio

$$
\frac{1}{f_{\rho}}: \frac{1}{f_{\omega}}: \frac{1}{f_{\phi}}=\sqrt{3}: S_{\omega} \sqrt{\frac{1}{3}}:-S_{\phi} \sqrt{\frac{2}{3}} .
$$

It allows us to express unambiguous formulas for the $f^{F}, f^{D}, f^{S}$ coupling constants which do not depend on the choice of the $\omega-\phi$ mixing

$$
\begin{aligned}
& f^{F}=\frac{1}{2}\left[\frac{f_{\rho N N}}{f_{\rho}}\left|f_{\rho}\right|+\sqrt{3}\left(\frac{f_{\phi N N}}{f_{\phi}}\left|f_{\phi}\right| \cos \theta+\frac{f_{\omega N N}}{f_{\omega}}\left|f_{\omega}\right| \sin \theta\right)\right] \\
& f^{D}=\frac{1}{2}\left[3 \frac{f_{\rho N N}}{f_{\rho}}\left|f_{\rho}\right|-\sqrt{3}\left(\frac{f_{\phi N N}}{f_{\phi}}\left|f_{\phi}\right| \cos \theta+\frac{f_{\omega N N}}{f_{\omega}}\left|f_{\omega}\right| \sin \theta\right)\right] \\
& f^{S}=\sqrt{2}\left(\frac{f_{\omega N N}}{f_{\omega}}\left|f_{\omega}\right| \cos \theta-\frac{f_{\phi N N}}{f_{\phi}}\left|f_{\phi}\right| \sin \theta\right) .
\end{aligned}
$$

\section{Acknowledgments}

This work was supported by Slovak Grant Agency for Science VEGA, Grant No. 2/0153/17 and Development Agency APVV, Grant No. APVV-0463-12.

\section{References}

[1] M. Gourdin, Unitary symmetries and their application to high energy physics, North-Holland (1967) .

[2] F. E. Close, An introduction to quarks and partons, Academic Press (1979) .

[3] S. Gasiorowicz, Elementary particle physics, Springer-Verlag (1966) .

[4] C. Adamuscin, E. Bartos, S. Dubnicka and A. Z. Dubnickova, Numerical values of $f^{F}, f^{D}, f^{S}$ coupling constants in $S U(3)$ invariant interaction Lagrangian of vector-meson nonet with $1 / 2^{+}$octet baryons, Phys. Rev. C93 (2016) 055208.

[5] N. M. Kroll, T. D. Lee and B. Zumino, Neutral vector mesons and the hadronic electromagnetic current, Phys. Rev. 157 (1967) 1376. 